Case Report

\title{
Early Posterior Rectus Sheath Hernia Recurrence After Open Incisional Hernia Repair with Retrorectus Mesh Placement
}

\author{
Grigoriy V. Klimovich ${ }^{\dagger}$, Randal Zhou ${ }^{\dagger, *}$, Kurt E. Roberts \\ Section of Gastrointestinal Surgery, Yale University, School of Medicine, New Haven, CT, USA
}

\section{Article history:}

Received: July 12, 2019

Revised: August 22, 2020

Accepted: August 22, 2020

$\dagger$ Grigoriy V Klimovich and Randal Zhou contributed equally as co-first authors.

\section{${ }^{*}$ Corresponding Author:}

Randal Zhou

Section of Gastrointestinal Surgery, Yale University, School of Medicine, 40 Temple

Street Suite 7B New Haven, CT 06510 USA

E-mail Randalzhou@gmail.com

\section{ORCID}

Randal Zhou

https://orcid.org/0000-0001-6713-4933

\section{ABSTRACT}

A 51-year-old female underwent recurrent open incisional hernia repair with retrorectus mesh placement. Early in the post-operative course, she developed a hernia reoccurrence secondary to breakdown of the staple line, at the level of the posterior rectus sheath, resulting in a small bowel obstruction. This hernia could not be felt upon physical examination but was detected by imaging. The patient was promptly taken to the operating room for laparoscopic reduction of the incarcerated loop of small intestine, along with laparoscopic repair of the posterior rectus sheath defect. It is critical for surgeons to recognize the possibility of a staple line breakdown at the level of posterior rectus sheath early on in the diagnosis which would prompt urgent surgical intervention in the setting of a bowel obstruction.

Keywords: hernia repair, intestinal obstruction, laparoscopy

\section{Introduction}

The reported incidence of incisional hernia after a laparotomy is $22.4 \%$ at the 3 -year mark [1]. Depending on the patient's risk factors, the actual incidence of incisional hernia can be higher or lower. Patients may be asymptomatic or present with abdominal pain, discomfort, disfigurement, or bowel obstruction. The hernias can be repaired laparoscopically, robotically, or in open surgery. An open approach is generally required for large incisional hernia repairs. Possible postoperative complications following an open incisional hernia repair include wound infections, bowel obstructions, and seroma or hematoma formations. In this study, a case of an early posterior rectus sheath hernia recurrence after an open retrorectus incisional hernia repair is presented. In this case, the recurrent hernia presented with a small bowel obstruction.

\section{Case Report}

A 51-year-old female originally presented with a $5 \times 5 \mathrm{~cm}$ midline incisional hernia (Figure 1). She had a surgical history of open ovarian surgery, laparoscopic sleeve gastrectomy, and a previous laparoscopic incisional hernia repair with an underlay polyester mesh. Her body mass index decreased from $40.2 \mathrm{~kg} / \mathrm{m}^{2}$ to $33 \mathrm{~kg} / \mathrm{m}^{2}$ after bariatric surgery. The patient's incisional hernia was repaired in an open approach with retrorectus polypropylene mesh placement. The abdominal cavity was entered through the midline. Adhesions were lysed and the previously placed polyester mesh was removed. The retrorectus plane was developed. Endo GIA black reload staples with Tri-Staple technology (Medtronic, Inc., Dublin, Ireland) were used to separate the anterior and posterior rectus sheaths in cephalad and caudal directions with respect to the hernia defect. This created a space for the mesh placement. First, the posterior rectus sheath was closed, then the polypropylene mesh was placed into the retrorectus space, and finally the anterior rectus sheath and skin were closed. The patient was discharged on Post-Operative Day (POD) \# 3.

On POD \# 10, the patient presented at the Emergency Department with increasing abdominal pain, nausea and vomiting. The patient's vital signs were within normal limits 


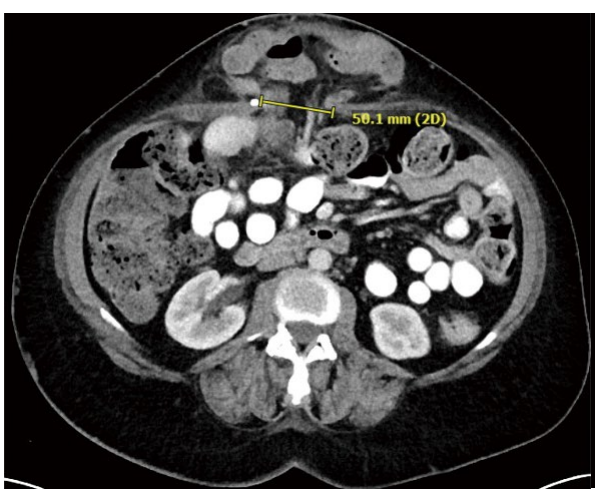

Figure 1. Incisional hernia with non-obstructing loops of bowel in the $5 \mathrm{~cm}$ hernia defect.

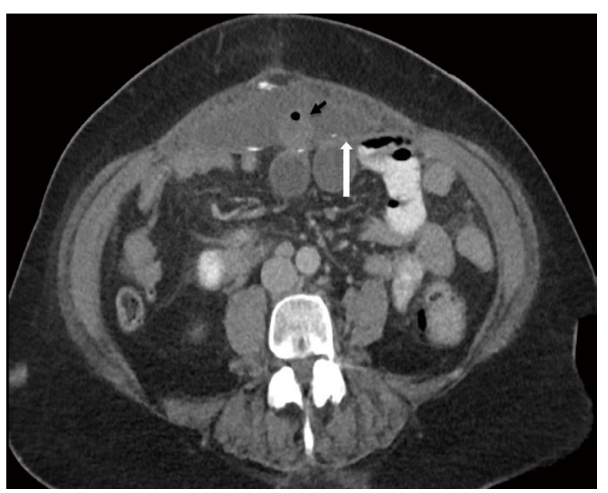

Figure 2. Transverse view, a loop of bowel (black arrow) herniating through the posterior rectus sheath (white arrow).

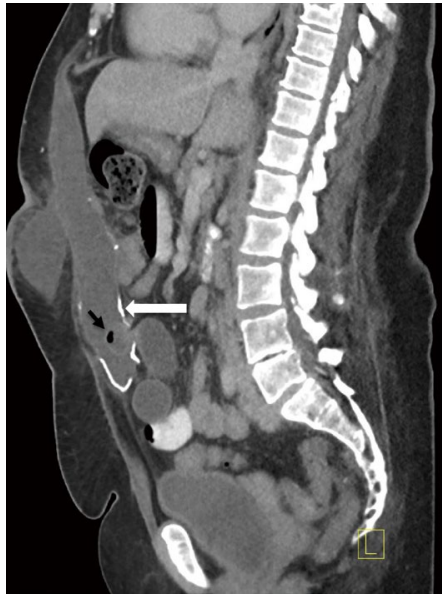

Figure 3. Sagittal view, a loop of bowel (black arrow) herniating through the posterior rectus sheath (white arrow). but her abdomen was noted to be tender to palpation. Her blood work was only significant for a mild leukocytosis (elevated white blood cell count of $13,000 / \mu \mathrm{L}$ ). The computerized tomography scan revealed that the patient had a loop of small intestine herniating though the posterior rectus sheath. The patient was immediately taken to the operating room for a laparoscopic repair of her posterior rectus sheath defect. Once the abdomen was entered laparoscopically, the loop of small intestine entering the $2.5 \mathrm{~cm}$ defect in the posterior rectus sheath was visualized. The proximal loops of bowel were dilated and the distal loops were decompressed. The defect resulted from a broken-down staple line that was used to separate the anterior and posterior rectus sheaths. The defect was closed laparoscopically with non-absorbable polyester suture (O-Ethibond); a total of 4 figure-of- 8 stitches were used. The patient was discharged on POD \#2. Figures 2 and 3 depict the herniation of small intestine through the posterior rectus sheath.

\section{Discussion}

A critical skill for all general and acute care surgeons is the ability to detect and treat hernias and complications of hernia repair. In the acute care setting, a patient may present with bowel obstruction due to an incisional hernia. Incisional hernias may be repaired laparoscopically, robotically or with an open surgery approach. Typically defects greater than $5 \mathrm{~cm}$ in size are better served with an open incisional hernia repair with retrorectus mesh placement. A retrorectus hernia repair is also known as a Rives-Stoppa repair. Defects that are greater than $10 \mathrm{~cm}$ may require a posterior component separation. It is the standard of care to use a permanent mesh for incisional hernia repair if there is no evidence of bowel compromise. The overall recurrence of hernias and complications after hernia repair is less than $10 \%[2-5]$.

Patients who present with evidence of bowel obstruction following incisional hernia repair need to be further evaluated by undergoing a computerized tomography scan. Obstruction can be due to either adhesions or breakdown of a posterior rectus sheath closure. This study is the first to report a postoperative small bowel obstruction resulting from posterior rectus sheath breakdown where an endo-GIA stapler was used for creating the retrorectus space. In this case, the loop of bowel was laparoscopically reduced and the $2.5 \mathrm{~cm}$ defect was closed with permanent stitches. No bowel resection was required. In patients with a history of a recent incisional hernia repair, surgeons should be aware of possible posterior rectus sheath breakdown even if no hernia is felt upon physical examination. Identification of a posterior rectus sheath hernia should prompt urgent surgical intervention.

\section{Conflicts of Interest}

The authors have no conflicts of interest to disclose.

\section{References}

[1] Fink C, Baumann P, Wente MN, Knebel P, Bruckner T, Ulrich A, et al. Incisional hernia rate 3 years after midline laparotomy. Br J Surg 2014;101(2):51-4. 
[2] Israelsson LA, Smedberg S, Montgomery A, Nordin P, Spangen L. Incisional hernia repair in Sweden 2002. Hernia 2006;10(3):258-61.

[3] Helgstrand F, Rosenburg J, Kehlet H. Jorgensen LN, Bisgaar T. Nationwide prospective study of outcomes after elective incisional hernia repair. J Am Coll Surg 2013;2016(2):217-28.
[4] McLanahan D, King LT, Weems C, Novotney M, Gibson K. Retrorectus prosthetic mesh repair of midline abdominal hernia. Am J Surg 1997;173(5):445-9.

[5] Martin-Dauce A, Noguerales F, Villeta R, Hernández P, Lozano O, Keller J et al. Modifications to Rives technique for midline incisional hernia repair. Hernia 2001;5(2):70-2. 